\title{
Análise da estrutura de comunidades arbóreas de uma floresta amazônica de Terra Firme aplicada ao manejo florestal
}

\author{
João Carlos Zenaide Oliveira ALVES ${ }^{1}$, Izildinha de Souza MIRANDA ${ }^{1,2}$
}

\begin{abstract}
RESUMO
Este trabalho teve o objetivo de identificar e descrever a estrutura das comunidades arbóreas de uma floresta de terra firme sob regime de manejo na Amazônia Oriental, localizada no município de Almeirim, Pará, Brasil. O levantamento florístico foi realizado em 1.400,30 ha de uma Unidade de Produção Anual (UPA), do Plano de Manejo Florestal da empresa ORSA Florestal. Todos os indivíduos com DAP $\geq 30 \mathrm{~cm}$ foram inventariados, registrando-se 77.834 árvores distribuídas em 57 famílias, 229 gêneros e 556 espécies. Oito comunidades foram identificadas por meio de uma analise de agrupamento, apresentando alta diversidade e equibilidade florística ( $H^{\prime}$ médio = 4,25 e J' médio = 0,75). As comunidades apresentaram 138 espécies comuns, 119 espécies de ocorrência exclusiva e 377 espécies raras, representadas por apenas um indivíduo. As espécies que mais se destacaram foram: Dinizia excelsa, Vouacapoua americana, Goupia glabra, Mouriri brachyanthera, Parinari excelsa, Manilkara bidentada, Tachigalia mymecophyla e Licania micrantha. Algumas espécies de valor comercial apresentaram variaçôes importantes na densidade, sugerindo risco de extinção em comunidades onde as espécies apresentam densidade muito baixa. Sugerimos que os planos de manejo considerem as várias comunidades ecológicas encontradas nas UPAs evitando assim variaçóes significativas, causadas pela exploração e seus impactos, na composição florística e estrutura das comunidades existentes.
\end{abstract}

PALAVRAS-CHAVE: Análise de agrupamento, Amazônia, Diversidade, Florística.

\section{Analysis of the structure of tree communities of a amazonian forest applied to management}

\begin{abstract}
To demonstrate the importance of the ecological concept for forest management, the object of this work was to identify and describe the structure of tree communities of a tropical forest under management in the Eastern Amazonia, located in Almeirim municipality, in the state of Pará, Brazil. The floristic survey was undertaken in a terra-firme tropical forest of 1,400.30 hectares. All individuals with $\mathrm{DBH} \geq 30 \mathrm{~cm}$ were inventoried, registering 77,834 trees distributed in 57 families, 229 genera and 556 species. Eight communities were identified, presenting high diversity and floristic equitability $\left(\mathrm{H}^{\prime}=4.25\right.$ average and J' $=0.75$ average). The communities presented 138 common species, 119 species of exclusive occurrence and 377 rare species, represented by only one individual. The most remarkable species found in the area were: Dinizia excelsa, Vouacapoua americana, Goupia glabra, Mouriri brachyanthera, Parinari excelsa, Manilkara bidentada, Tachigali mymecophyla and Licania micrantha. The communities presented significant variations between the averages of the main floristic parameters. Some species of commercial value had important variations in the density, suggesting extinction risk in communities where they present very low density. We suggest that all sustainable forest management plans consider the several ecological communities found in the Annual Production Unit (UPA) thus avoiding significant variations, caused by exploration and their impacts, in the floristic composition and structure of these communities.
\end{abstract}

KEYWORDS: Cluster, Amazonia, Density, Floristic.

1 Programa de Mestrado em Ciências Florestais, Universidade Federal Rural da Amazônia, CP 917, CEP 66077-530, Belém-PA

2 Autor para correspondência: e-mail: izildinha.miranda@ufra.edu.br 


\section{INTRODUÇÃO}

A manutenção da diversidade tanto de espécies como de habitats, é fundamental para o sucesso de empreendimentos florestais na Amazônia. Para isso, existe a necessidade de considerar a diversidade de habitats, as diferenças biogeográficas (Tuomisto et al., 2003) e o conhecimento dos processos ecológicos e seus agentes (Phillips et al., 2003a; Schulman, 2003; Lewis et al., 2004) na elaboração e condução de planos de manejo das florestas naturais.

Entretanto, os mecanismos de sustentação dos ecossistemas manejados, ainda não são adequadamente abordados pelos manejados e nem pela legislação florestal brasileira em vigor, visto que vários aspectos ecológicos fundamentais são desconsiderados nos planos de manejo. A Instrução Normativa $\mathrm{N}^{\circ} 4$ (Diário Oficial da União, No45 - Seção 1, 7 de março de 2002) estabelece critérios para manutenção da capacidade produtiva das florestas em longo prazo, tais como o controle do volume de madeira extraído por hectare, o ciclo de corte e algumas medidas silviculturais baseadas nas áreas das unidades de produção anual (UPA) e não em áreas com condiçōes ambientais uniformes, que corresponderiam a comunidades ecológicas, com suas propriedades coletivas e emergentes estabelecidas dentro dos padrôes e processos ecológicos conhecidos para as florestas tropicais.

Os aspectos ecológicos têm ganhado importância dentro do manejo de florestas naturais. A atividade de manejo tem procurado estabelecer práticas silviculturais mais adequadas aos processos ecológicos (Buschbacher, 1990; Rodriguez, 1998; Uhl \& Vieira, 1989), especialmente aqueles que podem afetar o crescimento ou a viabilidade das populações (Noris $\&$ McCulloch, 2003).

Conhecer as características e propriedades das diferentes comunidades vegetais, que formam essas florestas, poderá produzir resultados mais eficientes no manejo florestal, evitando mudanças significativas na composição florística e estrutura dessas vegetaçôes, mantendo o ambiente mais estável e preservando sua identidade ecológica (Oliver \& Larson, 1996).

O limite de uma comunidade é muito difícil de estabelecer, especialmente em florestas contínuas, onde áreas de transição não são visíveis. Vários autores discutem essa dificuldade (Condit et al., 2002; Phillips et al., 2003b; Tuomisto et al., 2003) e métodos de análises multivariadas têm sido utilizados para agrupar diversas amostras ou áreas revelando características estruturais específicas de cada grupo, considerados em geral como uma comunidade (Goodall, 1954; Greig-Smith, 1983). No entanto, o inverso desse raciocínio não tem sido explorado. Ou seja, separar, em um grande espaço contínuo, as diferentes comunidades existentes.
Nas florestas tropicais úmidas da Amazônia a alta diversidade de espécies existente está associada à alta diversidade de habitats e a grande especialização das espécies na utilização dos mesmos, resultando num grande número de espécies consideradas localmente raras (Hubbell \& Foster, 1986; Kochummen et al., 1990; Lieberman \& Lieberman, 1994) e na baixa similaridade florística entre parcelas próximas (Amaral et al., 2000; Campbell et al., 1986; Gentry, 1988). A dissimilaridade entre as comunidades vegetais na Amazônia também está associada, em parte, às variaçōes na topografia e nas características edáficas (Guillaumet, 1987; Gentry, 1988; Tuomisto et al., 1995).

Nesse contexto, este trabalho teve por objetivo identificar as diferentes comunidades arbóreas de uma floresta sob regime de manejo na Amazônia Oriental, descrever suas estruturas e analisar as variaçôes na densidade de algumas espécies de interesse comercial entre as comunidades identificadas.

\section{MATERIAL E MÉTODOS}

\section{ÁREA DE ESTUDO}

O estudo foi realizado em uma área de floresta primária do projeto de manejo da empresa Orsa Florestal, situada no vale do rio Arraiolos, afluente pela margem direita do rio Jari, município de Almeirim, estado do Pará, Brasil, entre as latitudes $0^{\circ} 27^{\prime}$ e $1^{\circ} 30^{\prime} \mathrm{S}$, e longitudes de $51^{\circ} 40^{\prime}$ e $53^{\circ}$ 20 ' W.

Os solos predominantes são os latossolos amarelos e os podzólicos vermelho-amarelos. Em menores intensidades ocorrem os plintossolos e os cambissolos. Os solos sob floresta de terra firme variam quanto aos teores de areia, argila e silte, e à maior ou menor presença de cascalho. Os solos sob floresta primária no vale do rio Jari não são uniformes, e apresentam variações não apenas com relação aos nutrientes, mas também na parte física, a qual é relacionada com o regime hídrico local (Jari, 2000).

O clima da região é do tipo Amw de Köppen, caracterizado como quente e úmido e apresenta duas estaçōes bem definidas: uma chuvosa, de janeiro a julho e outra seca, de agosto a dezembro. A temperatura média anual é de $26^{\circ} \mathrm{C} \pm 2^{\circ}$ e a precipitação pluviométrica média anual é de $2.115 \mathrm{~mm}$.

A vegetação da área estudada é composta por algumas fisionomias, destacando-se na parte sul os campos inundáveis (várzeas), matas de igapó ao longo dos rios, vegetação de cerrado e principalmente floresta de terra firme, dominando praticamente toda a paisagem.

\section{MÉTODOS DE CAMPO E DE ANÁLISES}

O estudo foi realizado na Unidade de Produção Anual $\mathrm{n}^{\circ} 1$ (UPA), da Unidade de Manejo $\mathrm{n}^{\circ} 1$ (UM), com 2.280 hectares. Essa área foi dividida em 228 Unidades de Trabalho 
(UTs), de 10 hectares cada ( $400 \times 250 \mathrm{~m}$ ). Dessas, apenas 195 UTs foram parcialmente ou totalmente inventariadas por não apresentarem restrições operacionais e ambientais, tais como relevo acidentado, áreas alagadas ou sujeitas a alagamento no período chuvoso e áreas de preservação permanente.

O inventário florestal foi realizado em 2001 contemplando $100 \%$ dos indivíduos com diâmetro a $1,3 \mathrm{~m}$ de altura (DAP) maior ou igual a $30 \mathrm{~cm}$, que estavam presentes nas 195 UTs estudadas. Foram medidos os DAP e identificado todos os indivíduos. A identificação botânica foi realizada por parabotânicos da empresa Orsa Florestal através da comparação das exsicatas coletadas junto ao Herbário ou Xiloteca da empresa.

A identificação das comunidades foi realizada através de uma análise de agrupamento utilizando Phi-quadrado como medida de distância entre as abundâncias das espécies nas UTs e o método de Ward para formação dos grupos (Ward, 1963; Reynolds, 1977). Esse método valoriza a variância da abundância das espécies nos grupos.

A composição florística foi analisada com base nos números de indivíduos, espécies, gêneros e famílias. A diversidade florística foi calculada pelo índice de ShannonWeiner $\left(\mathrm{H}^{\prime}=-\sum \mathrm{n}_{\mathrm{i}} \operatorname{Ln}\left(\mathrm{n}_{\mathrm{i}}\right)\right)$ e pela equibilidade $\left(\mathrm{J}=\mathrm{H}^{\prime}\right.$. $\operatorname{Ln}(S)^{-1}$ segundo (Magurran, 1988), onde $n_{i}$ é o número de indivíduos da espécie i, Ln é o logaritmo neperiano e $S$ é o número de espécies.

A estrutura horizontal foi descrita através dos parâmetros de abundância, dominância e freqüência, segundo Lamprecht (1964). O índice de valor de importância (IVI) das espécies foi obtido através do somatório dos valores relativos de abundância, dominância e freqüência (Finol, 1971). Os principais parâmetros estruturais apresentados pelas respectivas UTs das comunidades identificadas na análise de agrupamento, tiveram suas médias testadas pelo teste t a 5\% de probabilidade.

O programa ArcView/ESRI (Sistema de Informações Geográficas) foi utilizado para subdividir as UTs em parcelas de 20 por 25 metros $\left(500 \mathrm{~m}^{2}\right)$, totalizando 28.006 parcelas, que foram utilizadas para a determinação da freqüência das espécies com base na ocorrência. Este programa também foi utilizado para a manipulação do banco de dados e geração do mapa.

\section{RESULTADOS}

As 195 UTs inventariadas possuem área total de 1.400,30 ha, na qual foram encontrados 77.834 indivíduos pertencentes a 57 famílias, 229 gêneros e 556 espécies. As dezoito espécies mais abundantes (3,2\% do numero total de espécies), presentes na UPA, foram responsáveis por metade $(50,3 \%)$ do número total de indivíduos (Tabela 1).
Tabela 1 - Espécies mais abundantes presentes na UPA 1 estudadas em Almeirim (PA).

\begin{tabular}{llll}
\hline Família & Nome Botânico & No Ind. & \% \\
\hline Celastraceae & Goupia glabra Aublet & 4305 & 5,53 \\
Sapotaceae & Manilkara bidentada (A. DC.) A. Chev. & 2946 & 3,78 \\
\hline Chrysobalanaceae & Licania micrantha Sagote Mig & 2861 & 3,68 \\
\hline Mimosaceae & Dinizia excelsa Ducke & 2805 & 3,60 \\
\hline Caesalpiniaceae & Vouacapoua americana Aublet & 2670 & 3,43 \\
\hline Vochysiaceae & Qualea paraensis Ducke & 2631 & 3,38 \\
\hline Chrysobalanaceae & Parinari excelsa Sabine & 2468 & 3,17 \\
Melastomataceae & Mouriri brachyanthera Ducke & 2161 & 2,78 \\
\hline Caesalpiniaceae & Tachigalia myrmecophyla Ducke & 1984 & 2,55 \\
Vochysiaceae & Vochysia obscura Warm. & 1876 & 2,41 \\
Vochysiaceae & Qualea albiflora Warm. & 1793 & 2,30 \\
\hline Sapotaceae & Manilkara huberi Stand. & 1761 & 2,26 \\
\hline Humiriaceae & Vantanea parviflora Lam. & 1711 & 2,20 \\
\hline Sapotaceae & Nemaluma anomalum Pires & 1674 & 2,15 \\
\hline Olacaceae & Minquartia guianensis Aublet & 1602 & 2,06 \\
\hline Rubiaceae & Chimarrhis turbinata DC. & 1423 & 1,83 \\
\hline Fabaceae & Bowdichia nitida Bentham & 1309 & 1,68 \\
\hline Caesalpiniaceae & Tachigalia alba Ducke & 1161 & 1,49 \\
\hline Totais & & 39141 & 50,3 \\
\hline & & & \\
\hline & & &
\end{tabular}

A análise de agrupamento permitiu a classificação das 195 UTs em oito comunidades florestais distintas, que aparentemente segue um gradiente ambiental bastante influenciado pela topografia. A distribuição geográfica revela um gradiente ambiental que vai do platô, na região da Serra de Almeirim (cerca de $250 \mathrm{~m}$ de altitude), para a margem do rio Arraiolos (cerca de $30 \mathrm{~m}$ de altitude) e ao longo do vale do Arraiolos, no sentido jusante, até as proximidades do seu encontro com o rio Amazonas, onde a floresta de terra-firme dá lugar a uma zona de transição para floresta de várzea, fora dos limites da área de estudo (Figura 1). As comunidades foram denominadas pelo nome vulgar das duas principais espécies.

As comunidades apresentaram tamanhos bem variados, média de 175, 04 ha e coeficiente de variação (CV) de 49,88\% (Tabela 2 e Figura 1).

O número de famílias e gêneros apresentados pelas comunidades foram bem similares, médias em torno de 44,25 $(\mathrm{CV}=11,12 \%)$ e $146,88(\mathrm{CV}=13,51 \%)$ respectivamente.

Riqueza, densidade, dominância e diversidade de Shannon apresentaram diferenças significativas entre as comunidades (Tabela 2). A comunidade Angelim-Muiraúba, por exemplo, utilizando-se a riqueza como parâmetro, diverge estatisticamente das comunidades Acapu-Angelim e AngelimCupiúba, assim como, a comunidade Cupiúba-Maparajuba, empregando-se o índice de Shannon, diverge das comunidades Acapu-Angelim e Acapu-Cupiúba. Várias outras comparaçōes apresentaram diferenças significativas (Tabela 2). 
Tabela 2 - Riqueza e diversidade das comunidades florestais estudadas em Almeirim (PA). Número de indivíduos ( $\mathrm{N}^{0}$ Ind.), número de indivíduos por hectare ( $N^{0}$ Ind ha-1), dominância $\left(\operatorname{Dom}\left(\mathrm{m}^{2}\right.\right.$ ha-1 $)$ ), índice de Shannon (H'), equibilidade (J'), número de parcelas de 20x25m(№ Parcelas (20x25)) e tamanho da área das comunidade em hectares (ha), CV\% - Coeficiente de variação.

\begin{tabular}{|c|c|c|c|c|c|c|c|c|c|c|}
\hline Comunidades & Famílias & Gêneros & Riqueza & $N^{\circ}$ Ind. & $\mathrm{N}^{\circ}$ Ind ha-1 & $\begin{array}{l}\text { Dom } \\
\left(m^{2} \mathrm{ha}^{-1}\right)\end{array}$ & $H^{\prime}$ & $J^{\prime}$ & $\begin{array}{l}N^{\circ} \text { Parcelas } \\
(20 \times 25 \mathrm{~m})\end{array}$ & Área (ha) \\
\hline 1 - Angelim Muiraúba & 43 & 150 & $303 a$ & $12774 \mathrm{a}$ & $53,80 \mathrm{a}$ & $10,78 \mathrm{a}$ & $4,32 \mathrm{a}$ & 0,76 & 4749 & 237,45 \\
\hline 2 - Angelim Parinari & 41 & 142 & $285 a b$ & $8555 a b$ & $55,26 a b$ & $13,65 b$ & $4,22 \mathrm{ab}$ & 0,75 & 3096 & 154,80 \\
\hline 3-Acapu Angelim & 43 & 138 & $274 c$ & $6971 c$ & $54,76 a b c$ & $12,59 \mathrm{c}$ & $4,14 \mathrm{c}$ & 0,74 & 2546 & 127,30 \\
\hline 4 - Angelim Cupiúba & 53 & 177 & $404 d$ & $12883 \mathrm{abd}$ & $57,35 \mathrm{bcd}$ & $13,27 \mathrm{bcd}$ & $4,47 d$ & 0,75 & 4493 & 224,65 \\
\hline 5-Acapu Cupiúba & 40 & 128 & 241 abce & 3802 abcde & $62,58 \mathrm{de}$ & 12,99 bcde & 3,83 ce & 0,70 & 1215 & 60,75 \\
\hline 6 - Cupiúba Maparajuba & 41 & 131 & 246 abdef & 9389 bdef & $54,86 \mathrm{abcdf}$ & 9,93 af & 4,23 abdf & 0,77 & 3423 & 171,15 \\
\hline 7 - Acapu Tachi preto & 42 & 132 & 255 abceg & $5228 \mathrm{cg}$ & $56,37 \mathrm{abcdfg}$ & $11,23 \mathrm{ag}$ & 4,31 abfg & 0,78 & 1855 & 92,75 \\
\hline 8 - Cupiúba Cariperana & 51 & 177 & 379 abef & 18232 abce & $55,01 \mathrm{abcdfg}$ & 10,85 afg & $4,51 \mathrm{df}$ & 0,76 & 6629 & 331,45 \\
\hline Totais & 57 & 229 & 556 & 77834 & 55,58 & 11,91 & 4,33 & 0,75 & 28006 & 1400,30 \\
\hline Média & 44,25 & 146,88 & 298,38 & 9729,25 & 56,25 & 11,91 & 4,25 & 0,75 & 3500,75 & 175,04 \\
\hline CV\% & 11,12 & 13,51 & 20,57 & 48,52 & 4,94 & 11,56 & 4,95 & 3,19 & 49,88 & 49,88 \\
\hline
\end{tabular}

Letras diferentes representam diferença significativa para o teste $t(p<0,05)$.

As comunidades apresentaram em média $24,8 \%$ de espécies comuns. As espécies comuns às oito comunidades (138 espécies) foram consideradas como as mais tolerantes às variações ambientais do local, por estarem distribuídas por toda a área estudada. Entre essas espécies, a menos abundante foi Inga sp. com 22 indivíduos e a mais abundante foi Goupia glabra com 4305 indivíduos.

De ocorrência exclusiva, foram encontradas 11 espécies (2\%) na comunidade Angelim-Muiraúba, 16 (2,9\%) na Angelim-Parinari, 10 (1,8\%) na Acapu-Angelim, 46 (8,3\%) na Angelim-Cupiúba, $6(1,1 \%)$ na Acapu-Cupiúba, 3 (0,5\%) na Cupiúba-Maparajuba, $3(0,5 \%)$ na Acapu-Tachi preto e 25 (4,5\%) na Cupiúba-Cariperana.

As comunidades apresentaram um número muito baixo de espécies (5\%) respondendo pela grande maioria dos indivíduos (52\%); O número de espécies (média de 12,8 espécies, $\mathrm{CV}=17,2 \%)$ com densidade absoluta $\geq 1$ ind.ha ${ }^{-1}$ é muito baixo. A maioria das espécies (média=285,6 e CV=21,3\%) possui densidade inferior a um indivíduo por hectare (Tabela 3). Do total de 556 espécies encontradas, 377 (67,8\%) espécies foram representadas por apenas 1 único indivíduo em pelo menos uma das oito comunidades identificadas. A abundância dessas 377 espécies, considerando toda a área da UPA1, varia de 1 a 2670 indivíduos (média $=28,5$ e CV = 517,1\%), mostrando que as espécies podem apresentar raridade e abundância ao mesmo tempo, em diferentes comunidades onde se apresenta. No geral, considerando toda a área da UPA1, 79 espécies $(14,2 \%)$ foram representadas por apenas um único indivíduo.

Três espécies se destacaram como muito importantes ecologicamente em várias comunidades ao mesmo tempo: Dinizia excelsa (Angelim vermelho), muito importante nas comunidades Angelim-Muiraúba (IVI=30,63), AngelimParinari (IVI=41,02), Acapu-Angelim $(I V I=28,52)$ e
Angelim-Cupiúba (IVI=29,27); Vouacapoua americana (Acapu) importante nas comunidades Acapu-Angelim (IVI=28,88), Acapu-Cupiúba (IVI=66,97) e AcapuTachi preto (IVI=34,39); e Goupia glabra (Cupiúba) nas comunidades Angelim-Cupiúba (IVI=21,76), Acapu-Cupiúba (IVI=14,37), Cupiúba-Maparajuba (IVI=29,27) e CupiúbaCariperana (IVI=19,92) (Tabela 4).

A análise da densidade de oito espécies de valor comercial, consideradas importantes ecologicamente nas comunidades, mostra variações significativas entre as comunidades (Tabela 5). Um dos exemplos extremos foi Vouacapoua americana (Acapu), apresentando alta densidade na comunidade AcapuCupiúba (16,4 ind.ha-1), enquanto que na comunidade Angelim-Muiraúba aparece com densidade igual a 0,004 ind. $\mathrm{ha}^{-1}$. Outras espécies também tiveram variaçōes importantes na densidade, mostrando comportamentos diferenciados quando há mudanças na associação entre as espécies (Tabela 5).

Tabela 3 - № de espécies por comunidade com densidade absoluta $<$ e $\geq$ 1 estudadas em Almeirim (PA).

\begin{tabular}{lll}
\hline & \multicolumn{2}{c}{$N^{0}$ de espécies com } \\
\cline { 2 - 3 } & $\mathrm{n}^{0}$ Ind./ha $<1$ & $\mathrm{n}^{0}$ Ind./ha $\geq 1$ \\
\hline 1 - Angelim Muiraúba & 289 & 14 \\
2 - Angelim Parinari & 270 & 15 \\
3 - Acapu Angelim & 262 & 12 \\
\hline 4 - Angelim Cupiúba & 388 & 16 \\
\hline 5 - Acapu Cupiúba & 228 & 13 \\
6 - Cupiúba Maparajuba & 236 & 10 \\
\hline 7 - Acapu Tachi preto & 243 & 12 \\
\hline 8 - Cupiúba Cariperana & 369 & 10 \\
\hline Média & 285,6 & 12,8 \\
\hline CV\% & 21,3 & 17,2 \\
\hline
\end{tabular}


Tabela 4 - Dados estruturais das dez principais espécies em valor de importância ecológica das comunidades estudadas em Almeirim (PA). Densidade (Ab ha-1), dominância (Dom(m2 ha-1)), abundância relativa (Ab r\%), dominância relativa (Dom r\%), freqüência relativa (Freq r\%), índice de valor de importância (IVI).

\begin{tabular}{|c|c|c|c|c|c|c|}
\hline Espécie & $\mathrm{Ab} / \mathrm{ha}$ & Dom $\left(m^{2}\right) / h a$ & $A b \_r \%$ & Dom_r \% & Freq_r \% & IVI \\
\hline \multicolumn{7}{|l|}{ Angelim Muiraúba } \\
\hline Dinizia excelsa Ducke & 3,559 & 1,915 & 6,61 & 17,76 & 6,25 & 30,63 \\
\hline Mouriri brachyanthera Ducke & 2,678 & 0,360 & 4,98 & 3,34 & 4,88 & 13,20 \\
\hline Nemaluma anomalum Pires & 2,586 & 0,388 & 4,81 & 3,60 & 4,65 & 13,06 \\
\hline Manilkara bidentada (A. DC.) A. Chev. & 2,506 & 0,369 & 4,66 & 3,42 & 4,35 & 12,43 \\
\hline Parinari excelsa Sabine & 2,312 & 0,426 & 4,30 & 3,95 & 4,15 & 12,40 \\
\hline Vochysia obscura Warm. & 2,110 & 0,358 & 3,92 & 3,32 & 3,69 & 10,93 \\
\hline Goupia glabra Aublet & 1,887 & 0,369 & 3,51 & 3,42 & 3,45 & 10,38 \\
\hline Mezilaurus lindaviana Mez & 1,546 & 0,393 & 2,87 & 3,64 & 2,79 & 9,31 \\
\hline Vantanea parviflora Lam. & 1,802 & 0,289 & 3,35 & 2,68 & 3,23 & 9,26 \\
\hline Qualea paraensis Ducke & 1,407 & 0,332 & 2,61 & 3,08 & 2,61 & 8,30 \\
\hline \multicolumn{7}{|l|}{ Angelim Parinari } \\
\hline Dinizia excelsa Ducke & 3,882 & 3,718 & 7,03 & 27,24 & 6,76 & 41,02 \\
\hline Parinari excelsa Sabine & 4,096 & 0,978 & 7,41 & 7,17 & 6,96 & 21,54 \\
\hline Mouriri brachyanthera Ducke & 3,798 & 0,548 & 6,87 & 4,01 & 6,38 & 17,26 \\
\hline Nemaluma anomalum Pires & 2,565 & 0,416 & 4,64 & 3,05 & 4,56 & 12,24 \\
\hline Manilkara huberi Stand. & 2,358 & 0,486 & 4,27 & 3,56 & 4,21 & 12,04 \\
\hline Goupia glabra Aublet & 2,235 & 0,496 & 4,04 & 3,63 & 3,93 & 11,61 \\
\hline Tachigali myrmecophyla Ducke & 2,164 & 0,370 & 3,92 & 2,71 & 3,81 & 10,44 \\
\hline Aniba squarensis Vatlimo Gil & 1,460 & 0,233 & 2,64 & 1,71 & 2,68 & 7,03 \\
\hline Sclerolobium melanocarpum Ducke & 1,273 & 0,305 & 2,30 & 2,24 & 2,30 & 6,84 \\
\hline Chimarrhis turbinata DC. & 1,202 & 0,233 & 2,17 & 1,71 & 2,21 & 6,10 \\
\hline \multicolumn{7}{|l|}{ Acapu Angelim } \\
\hline Vouacapoua americana Aublet & 6,104 & 1,114 & 11,15 & 8,84 & 8,89 & 28,88 \\
\hline Dinizia excelsa Ducke & 2,616 & 2,399 & 4,78 & 19,05 & 4,69 & 28,52 \\
\hline Eschweilera odorata Poepp. & 5,232 & 0,780 & 9,55 & 6,20 & 8,74 & 24,49 \\
\hline Tachigalia myrmecophyla Ducke & 2,506 & 0,503 & 4,58 & 3,99 & 4,63 & 13,20 \\
\hline Parinari excelsa Sabine & 1,822 & 0,410 & 3,33 & 3,25 & 3,39 & 9,98 \\
\hline Chimarrhis turbinata DC. & 1,736 & 0,411 & 3,17 & 3,27 & 3,25 & 9,69 \\
\hline Manilkara huberi Stand. & 1,626 & 0,373 & 2,97 & 2,97 & 3,01 & 8,94 \\
\hline Eschweilera paniculata Mori & 1,681 & 0,303 & 3,07 & 2,40 & 3,11 & 8,59 \\
\hline Goupia glabra Aublet & 1,367 & 0,293 & 2,50 & 2,33 & 2,56 & 7,38 \\
\hline Minquartia guianensis Aublet & 1,084 & 0,370 & 1,98 & 2,94 & 2,14 & 7,06 \\
\hline \multicolumn{7}{|l|}{ Angelim Cupiúba } \\
\hline Dinizia excelsa Ducke & 3,143 & 2,466 & 5,48 & 18,58 & 5,21 & 29,27 \\
\hline Goupia glabra Aublet & 4,327 & 0,930 & 7,54 & 7,01 & 7,20 & 21,76 \\
\hline Qualea paraensis Ducke & 2,524 & 0,668 & 4,40 & 5,04 & 4,22 & 13,65 \\
\hline Qualea albiflora Warm. & 2,297 & 0,632 & 4,01 & 4,77 & 3,95 & 12,72 \\
\hline Licania micrantha Sagote Mig & 1,870 & 0,255 & 3,26 & 1,92 & 3,16 & 8,34 \\
\hline Mouriri brachyanthera Ducke & 1,812 & 0,249 & 3,16 & 1,88 & 3,15 & 8,19 \\
\hline Tachigalia myrmecophyla Ducke & 1,447 & 0,292 & 2,52 & 2,20 & 2,49 & 7,21 \\
\hline Manilkara huberi Stand. & 1,358 & 0,290 & 2,37 & 2,19 & 2,35 & 6,90 \\
\hline Licania robusta Sagot & 1,456 & 0,208 & 2,54 & 1,57 & 2,50 & 6,61 \\
\hline Vochysia obscura Warm. & 1,398 & 0,226 & 2,44 & 1,70 & 2,41 & 6,55 \\
\hline
\end{tabular}


Tabela 4 - Continuação

\begin{tabular}{|c|c|c|c|c|c|c|}
\hline Espécie & $\mathrm{Ab} / \mathrm{ha}$ & $\operatorname{Dom}\left(\mathrm{m}^{2}\right) / \mathrm{ha}$ & Ab_r $\%$ & Dom_r \% & Freq_r \% & IVI \\
\hline \multicolumn{7}{|l|}{ Acapu Cupiúba } \\
\hline Vouacapoua americana Aublet & 16,428 & 2,963 & 26,25 & 22,81 & 17,91 & 66,97 \\
\hline Goupia glabra Aublet & 2,782 & 0,677 & 4,45 & 5,21 & 4,72 & 14,37 \\
\hline Licania micrantha Sagote Mig & 2,798 & 0,430 & 4,47 & 3,31 & 4,72 & 12,50 \\
\hline Chimarrhis turbinata DC. & 1,909 & 0,448 & 3,05 & 3,45 & 3,36 & 9,86 \\
\hline Minquartia guianensis Aublet & 1,695 & 0,524 & 2,71 & 4,03 & 2,93 & 9,67 \\
\hline Qualea paraensis Ducke & 1,827 & 0,450 & 2,92 & 3,47 & 3,15 & 9,53 \\
\hline Tachigalia myrmecophyla Ducke & 1,761 & 0,370 & 2,81 & 2,85 & 3,02 & 8,69 \\
\hline Manilkara huberi Stand. & 1,350 & 0,300 & 2,16 & 2,31 & 2,42 & 6,88 \\
\hline Laetia procera (Poepp.) Eich. & 1,449 & 0,239 & 2,31 & 1,84 & 2,51 & 6,66 \\
\hline Dinizia excelsa Ducke & 0,757 & 0,521 & 1,21 & 4,01 & 1,24 & 6,46 \\
\hline \multicolumn{7}{|l|}{ Cupiúba Maparajua } \\
\hline Goupia glabra Aublet & 5,229 & 1,058 & 9,53 & 10,65 & 9,08 & 29,27 \\
\hline Manilkara bidentada (A. DC.) A. Chev. & 4,020 & 0,639 & 7,33 & 6,43 & 6,68 & 20,44 \\
\hline Qualea paraensis Ducke & 2,583 & 0,662 & 4,71 & 6,66 & 4,68 & 16,05 \\
\hline Vochysia obscura Warm. & 2,583 & 0,405 & 4,71 & 4,08 & 4,53 & 13,32 \\
\hline Vantanea parviflora Lam. & 2,337 & 0,361 & 4,26 & 3,64 & 4,05 & 11,94 \\
\hline Qualea albiflora Warm. & 1,852 & 0,464 & 3,38 & 4,67 & 3,27 & 11,32 \\
\hline Licania micrantha Sagote Mig & 1,952 & 0,246 & 3,56 & 2,48 & 3,47 & 9,50 \\
\hline Tachigalia sp. & 1,151 & 0,146 & 2,10 & 1,47 & 2,12 & 5,69 \\
\hline Bowdichia nitida Bentham & 1,069 & 0,166 & 1,95 & 1,68 & 2,01 & 5,63 \\
\hline Mezilaurus lindaviana Mez & 0,935 & 0,224 & 1,70 & 2,26 & 1,67 & 5,63 \\
\hline \multicolumn{7}{|l|}{ Acapu Tachi preto } \\
\hline Vouacapoua americana Aublet & 7,439 & 1,188 & 13,20 & 10,58 & 10,62 & 34,39 \\
\hline Tachigalia myrmecophyla Ducke & 2,566 & 0,495 & 4,55 & 4,41 & 4,40 & 13,36 \\
\hline Licania micrantha Sagote Mig & 2,663 & 0,397 & 4,72 & 3,53 & 4,63 & 12,89 \\
\hline Dinizia excelsa Ducke & 1,197 & 0,841 & 2,12 & 7,49 & 2,12 & 11,73 \\
\hline Chimarrhis turbinata DC. & 2,092 & 0,456 & 3,71 & 4,06 & 3,77 & 11,54 \\
\hline Parinari excelsa Sabine & 2,070 & 0,447 & 3,67 & 3,98 & 3,60 & 11,25 \\
\hline Manilkara huberi Stand. & 1,941 & 0,450 & 3,44 & 4,00 & 3,33 & 10,78 \\
\hline Minquartia guianensis Aublet & 1,380 & 0,321 & 2,45 & 2,86 & 2,57 & 7,88 \\
\hline Qualea paraensis Ducke & 1,272 & 0,294 & 2,26 & 2,62 & 2,33 & 7,20 \\
\hline Eschweilera paniculata Mori & 1,445 & 0,243 & 2,56 & 2,16 & 2,41 & 7,13 \\
\hline \multicolumn{7}{|l|}{ Cupiúba Cariperana } \\
\hline Goupia glabra Aublet & 3,557 & 0,784 & 6,47 & 7,23 & 6,22 & 19,92 \\
\hline Licania micrantha Sagote Mig & 3,147 & 0,432 & 5,72 & 3,98 & 5,27 & 14,97 \\
\hline Manilkara bidentada (A. DC.) A. Chev. & 2,758 & 0,497 & 5,01 & 4,58 & 4,74 & 14,33 \\
\hline Qualea paraensis Ducke & 2,396 & 0,595 & 4,35 & 5,48 & 4,07 & 13,90 \\
\hline Minquartia guianensis Aublet & 1,798 & 0,520 & 3,27 & 4,80 & 3,19 & 11,26 \\
\hline Tachigalia alba Ducke & 1,656 & 0,350 & 3,01 & 3,23 & 3,00 & 9,24 \\
\hline Vochysia obscura Warm. & 1,448 & 0,246 & 2,63 & 2,26 & 2,58 & 7,48 \\
\hline Qualea albiflora Warm. & 1,029 & 0,285 & 1,87 & 2,63 & 1,84 & 6,34 \\
\hline Parinari excelsa Sabine & 1,110 & 0,214 & 2,02 & 1,98 & 1,97 & 5,96 \\
\hline Vantanea parviflora Lam. & 1,110 & 0,179 & 2,02 & 1,65 & 2,01 & 5,67 \\
\hline
\end{tabular}


Tabela 5 - Variação na densidade (Ind. ha-1) das oito principais espécies comerciais presentes nas comunidades estudadas em Almeirim (PA).

\begin{tabular}{|c|c|c|c|c|c|c|c|c|c|}
\hline Espécies comerciais & & Comunida & & & & & & & \\
\hline Nome Botânico & Nome Vulgar & $\begin{array}{l}\text { Angelim } \\
\text { Muiraúba }\end{array}$ & $\begin{array}{l}\text { Angelim } \\
\text { Parinari }\end{array}$ & $\begin{array}{l}\text { Acapu } \\
\text { Angelim }\end{array}$ & $\begin{array}{l}\text { Angelim } \\
\text { Cupiúba }\end{array}$ & $\begin{array}{l}\text { Acapu } \\
\text { Cupiúba }\end{array}$ & $\begin{array}{l}\text { Cupiúba } \\
\text { Maparajuba }\end{array}$ & $\begin{array}{l}\text { Acapu Tachi } \\
\text { preto }\end{array}$ & $\begin{array}{l}\text { Cupiúba } \\
\text { Cariperana }\end{array}$ \\
\hline Mouriri brachyanthera Ducke & Muiraúba & 2,678 & 3,798 & 0,990 & 1,812 & 1,053 & 0,684 & 0,162 & 0,628 \\
\hline Dinizia excelsa Ducke & $\begin{array}{l}\text { Angelim } \\
\text { vermelho }\end{array}$ & 3,559 & 3,882 & 2,616 & 3,143 & 0,757 & 0,643 & 1,197 & 0,160 \\
\hline $\begin{array}{l}\text { Vouacapoua americana } \\
\text { Aublet }\end{array}$ & Acapu & 0,004 & 0,000 & 6,104 & 0,396 & 16,428 & 0,204 & 7,439 & 0,241 \\
\hline Vochysia obscura Warm. & Quaruba rosa & 2,110 & 0,614 & 0,157 & 1,398 & 0,214 & 2,583 & 0,119 & 1,448 \\
\hline Manilkara huberi Stand. & Maçaranduba & 1,487 & 2,358 & 1,626 & 1,358 & 1,350 & 0,578 & 0,000 & 0,513 \\
\hline Minquartia guianensis Aublet & Acariquara & 0,691 & 1,027 & 1,084 & 0,935 & 1,695 & 0,608 & 1,380 & 1,798 \\
\hline Goupia glabra Aublet & Cupiúba & 1,887 & 2,235 & 1,367 & 4,327 & 2,782 & 5,229 & 1,315 & 3,557 \\
\hline Parinari excelsa Sabine & Parinari & 2,312 & 4,096 & 1,822 & 1,215 & 1,053 & 0,911 & 2,070 & 1,110 \\
\hline
\end{tabular}

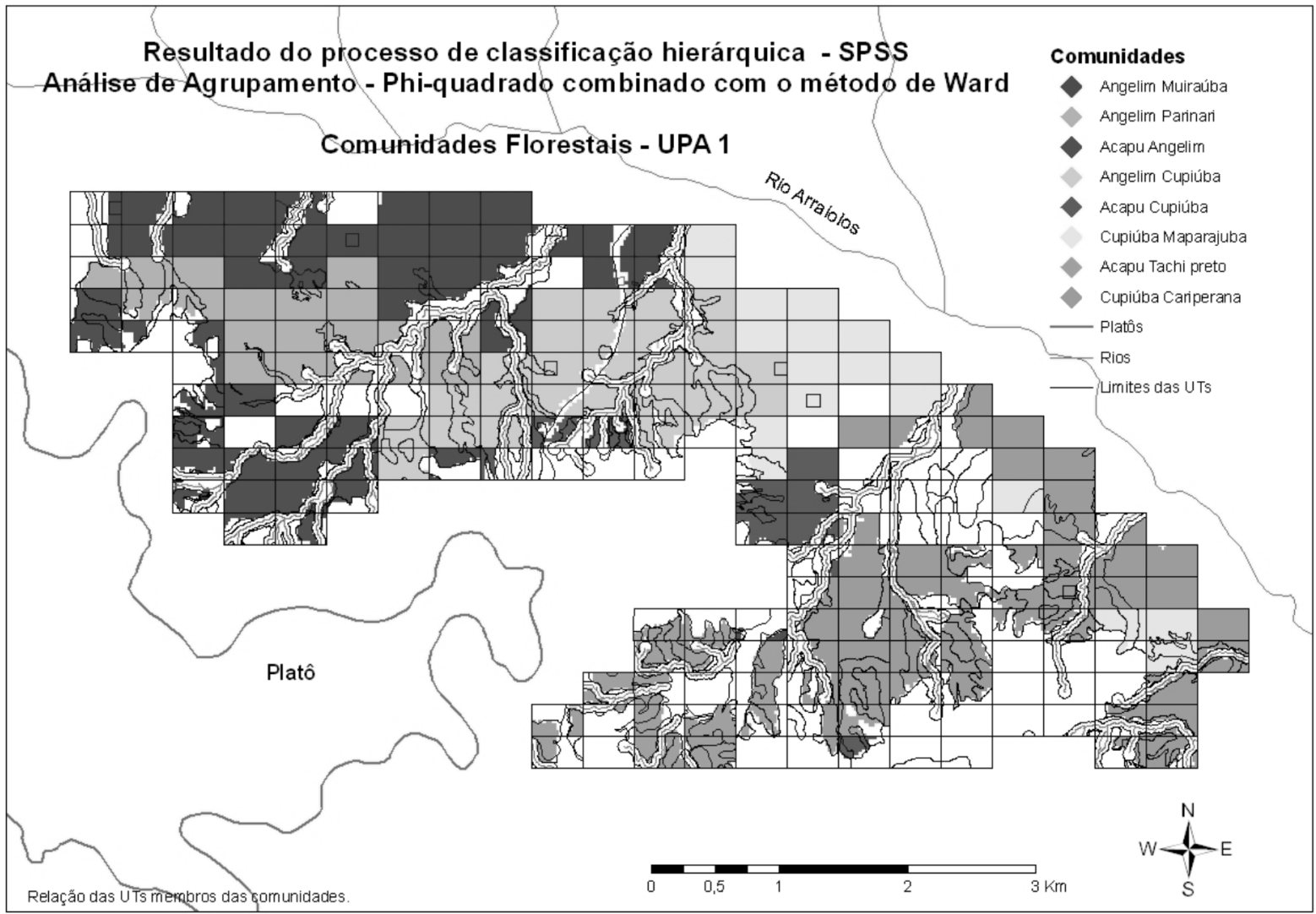

Figura 1 - Distribuição das comunidades na UPA 1, estudadas em Almeirim (PA).

\section{DISCUSSÃO}

A composição florística e estrutura horizontal dessas comunidades revelou vegetaçōes arbóreas extremamente ricas em número de famílias, gêneros e espécies, comparando-se com outros trabalhos desenvolvidos na Amazônia (Maciel et al., 2000; Oliveira \& Amaral, 2004; Pereira et al., 2005).
O índice de diversidade de Shannon-Weaver, para florestas tropicais amazônicas, normalmente varia entre 3,83 a 5,85, valores considerados altos para qualquer tipo de vegetação (Knight, 1975). Neste trabalho, os valores encontrados nas comunidades estudadas podem ser considerados altos, embora menores que o encontrado por Oliveira e Amaral (2004) na 
Estação Experimental ZF-2 do INPA em Manaus ( $\left.\mathrm{H}^{\prime}=5,01\right)$ e por Sist \& Saridan (1999) em Borneo, na Indonésia $\left(H^{\prime}=8,0\right)$. Deve-se considerar nesta comparação, que tanto Oliveira \& Amaral (2004) como Sist \& Saridan (1999), trabalharam com diâmetro mínimo de $10 \mathrm{~cm}$, aumentando bastante a chance de se obter um índice de diversidade superior ao deste estudo.

A alta diversidade de habitats e a especialização das espécies aos mesmos, explica o aparecimento de um grande número de espécies representadas por apenas 1, 2 ou 3 indivíduos (Hubbell \& Foster, 1986; Kochummen et al., 1990; Lieberman \& Lieberman, 1994). Patil \& Taillie (1982) referem-se à diversidade como uma média da raridade das espécies dentro da comunidade e consideram as medidas de diversidade como a probabilidade de encontros inter-específicos.

Estudos têm demonstrado que a riqueza de espécies está relacionada a diversos fatores ambientais, tais como, latitude, altitude, precipitação, nutrientes no solo, entre outros (Huston, 1980; Gentry, 1982), e que, existe correlação positiva entre a diversidade de espécies e a estabilidade dos sistemas florestais (Whittaker et al., 2001; Tilman et al., 2001). Sendo assim, em função dos elevados índices de diversidade e equibilidade apresentados pelas comunidades estudadas, pode-se considerá-las estáveis e independentes, fato que sugere uma maior consideração de suas existências nos atuais planos de manejo, que infelizmente, obedecendo à legislação em vigor para esse tipo de atividade, considera apenas a UPA como unidade produtiva, desprezando as variaçôes ecológicas existentes.

Vários trabalhos consideram espécies raras aquelas com densidade absoluta inferior a 1 ind.ha ${ }^{-1}$ (Almeida et al., 1993; Kageyama \& Gandara, 1994). Neste estudo foram encontradas apenas 17 espécies com densidade $\geq 1$, ou seja, por esse critério $96,9 \%$ das espécies são consideradas raras, portanto, teoricamente impedidas de serem utilizadas nos planos de manejo. O limite do número de indivíduos por ha como conceituação de raridade é relativo e depende da distribuição de abundância no ecossistema considerado para estudo (Kageyama \& Lepsch-Cunha, 2001).

Os dados mostrados por meio das comunidades revelam muito mais espécies raras (377 espécies), ou seja, representadas por apenas um indivíduo, do que mostrados por unidade de produção anual (UPA) (apenas 79 espécies). Segundo Whittaker (1972), em um gradiente de recursos, as espécies evoluem para ocupar diferentes posiçōes ao longo desse gradiente, conseqüentemente apresentando variações nos padrões de distribuição de abundância de acordo com a variação ambiental. Assim, as espécies apresentam raridade em determinadas locais e abundância em outras, conforme se observa nos resultados deste trabalho, ficando clara a necessidade de se planejar números diferentes de indivíduos a serem preservados para uma mesma espécie, em função dos diferentes graus de raridade apresentados pela espécie nas diferentes comunidades e não apenas numa área arbitrária, sem significado ecológico, como é a UPA, definida nos planos de manejo.

Este estudo revelou 120 espécies de ocorrência exclusiva apresentando baixas densidades, as quais foram consideradas especialistas em suas respectivas comunidades, por habitarem apenas em locais onde uma determinada associação de espécies e ou fatores ambientais permitem os seus estabelecimentos. Tais espécies atendem a pelo menos um dos três critérios (especificidade de habitats) proposto por Rabinowitz (1981), para avaliar os componentes de raridade das espécies. Alguns ecologistas e conservacionistas acreditam que certas propriedades das populaçôes as tornam mais vulneráveis à extinção. Uma delas é o tamanho da população, especialmente a raridade das espécies (Ricklefs, 2000).

As comunidades mostraram diferenças significativas entre a comparação das médias dos parâmetros estruturais analisados, corroborando com o resultado da análise de agrupamento. A presença de espécies comuns às comunidades confirma a estratégia da empresa em selecionar áreas relativamente homogêneas, do ponto de vista operacional, para fins de manejo. No entanto, do ponto de vista ecológico, as diferenças encontradas permitem caracterizar grupos de organismos coexistindo, expressando o caráter social das comunidades estudadas. Para Muniz et al. (1994), as espécies arbóreas que compõem a flora amazônica, coexistem em comunidades devido a uma similaridade aparente de exigências ecológicas, explicando com isso, o grande número de espécies comuns encontradas entre as comunidades.

As variaçōes, na densidade, apresentadas pelas espécies comerciais, nas diferentes comunidades, revela a importância de se considerar nos planos de manejo o conceito de comunidade florestal. A espécie Vouacapoua americana, pelas variaçōes apresentadas, demonstra claramente esta necessidade. O mesmo acontece com outras espécies comerciais, mostrando a importância desse nível de conhecimento para o manejo de florestas naturais. A inexistência de informaçôes sobre o comportamento das espécies comerciais nas diferentes comunidades, inclusive dos estoques de regeneração natural dessas espécies, aumenta significativamente o risco da exclusão das mesmas nas comunidades onde apresentam baixa densidade, podendo inclusive dar início a processos ecológicos indesejáveis com a diminuição da diversidade local.

$\mathrm{O}$ aparecimento de um grande número de espécies de baixa abundância foi certamente favorecido pelo registro, na coleta de dados, de $100 \%$ dos indivíduos com $\mathrm{DAP} \geq 30 \mathrm{~cm}$. Se não fosse aplicado o censo e sim um método de amostragem, a probabilidade do aparecimento de todas as espécies de baixa abundância seria pequena, levando ao aparecimento de um número menor de espécies nessa situação (Preston, 1948). 


\section{CONCLUSÕES}

A composição florística, estrutura e índices de diversidades apresentados pelas comunidades estudadas, permite classificálas como áreas de altíssimo valor biológico, representativas das florestas naturais (não perturbadas) de terra firme da Amazônia.

As espécies apresentam raridade em determinadas locais e abundância em outras, confirmando a necessidade de planejar, para o manejo florestal, números diferentes de indivíduos a serem preservados para uma mesma espécie em diferentes locais da UPA.

As variações na densidade, apresentadas pelas espécies comerciais, nas diferentes comunidades, confirma a importância de se considerar nos planos de manejo o conceito de comunidade florestal, especialmente para minimizar os impactos nas espécies mais especialistas em alguns habitats.

\section{BIBLIOGRAFIA CITADA}

Almeida, S.S.; Lisboa, P.L.B.; Silva, A.S.L. 1993. Diversidade florística de uma comunidade arbórea na Estação Científica Ferreira Penna, em Caxiuanã (Pará). Bol. Mus. Para. Emílio Goeldi, ser. Bot., 9(1): 99-105.

Amaral, I.L.; Matos, F.D.A.; Lima, J. 2000. Composição florística e estrutural de um hectare de floresta densa de terra firme no Rio Uatumã, Amazônia, Brasil. Acta Amazonica, 30: 377-392.

Buschbacher, R.J. 1990. Natural forest management in the humid tropic: ecological, social, and economic considerations. Ambio, 19(5): 253-258.

Campell, D.C.; Daly, D.C.; Prance, G.T.; Maciel, U.N. 1986. Quantitative ecological inventary of terra firme and varzea tropical forest on the rio Xingu, Brazilian Amazon. Brittonia, 38(4): 369-393.

Condit, R.; Pitman, N.; Leigh, E.G.; Chave, J.; Terborgh, J.; Foster, R.B. 2002. Beta-diversity in tropical forest trees. Science, 295: 666-669.

Finol, U.H. 1971. Nuevos parametros a considerarse em el análisis estructural de las selvas virgines tropicales. Rev. For. Venez., 14(21): 29-42.

Gentry, A.H. 1982. Patterns of neotropical plant species diversity. Evolutionary Biology, 15: 1-84.

Gentry, A. H. 1988. Changes in plant community diversity and floristic composition on environmental and geographical gradients. Ann. Missouri Bot. Gard., 75: 1-34.

Goodall, D.W. 1954. Objective methods in the classification of vegetation. III. An essay in the use of factor analysis. Aust. J. Bot., 2: 304-324.

Greig-Smith, P. 1983. Quantitative plant ecology. Blackwell, Oxford, 359pp.

Guillaumet, J.L. 1987. Some structural and floristic aspect of the forest. Experientia, 43(3): 241-251.

Hubbell, S.P.; Foster, R.B. 1986. Commonness and rarity in a neotropical forest: implications for tropical tree conservation.
In: Soulé, M.E. (ed.) Conservation biology: the science of scarcity and diversity. Sinauer, Massachusetts. p.205-231.

Huston, M.A. 1980. Soil nutrients and tree species richness in Costa Rican forests. Journal of Biogeography, 7: 147-157.

Jari. 2000. Plano de Manejo Florestal Sustentável. Jari Celulose S.A., Monte Dourado. (Documentos Técnicos).

Kageyama, P.Y.; Gandara, F.B. 1994. Dinâmica de populações de espécies arbóreas: implicações para o manejo e a conservação. Aciesp, 2: 1-9.

Kageyama, P.; Lepsch-Cunha, N.M. 2001. Singularidade da biodiversidade nos trópicos. In: Garay, I.; Dias, B. (eds.) Conservação da Biodiversidade em ecossistemas tropicais: Avanços conceituais e revisão de novas metodologias de avaliação $e$ monitoramento. São Paulo: Editora Vozes, 432pp.

Knight, D.H. 1975. A phytosociological analysis of species-rich tropical forest on Barro Colorado Island, Panama. Ecological Monographs, 45: 259-28.

Kochummen, K.M.; Lafrankie, J.V.; Manokaran, N. 1990. Floristic composition of Pasoh Forest Reserve, a lowland rain forest in Peninsular Malaysia. Journal of Tropical Forest Science, 3: 1-13.

Lamprecht, H. 1964. Ensayo sobre la estrutura floristica de la parte sur - oriental del bosque universitario "El caimital", Estado Barinas. Rev. For. Venezolana, 7(10/11): 77-119.

Lewis, S.L.; Malhi, Y.; Phillips, O.L. 2004. Fingerprinting the impacts of global change on tropical forests. Phil. Trans. R. Soc. Lond., 359: 437-462.

Lieberman, M.; Lieberman, D. 1994. Patterns of density and dispersion of forest trees. In: McDade, L.A., Bawa, K.S., Hespenheide, H.A., Hartshorn, G.S. (eds.), La Selva: Ecology and Natural History of a Neotropical Rain Forest. University of Chicago Press, Chicago. p.106-119.

Maciel, M.N.M.; Queiroz, W.T.; Oliveira, F.A. 2000. Parâmetros fitossociológicos de uma floresta tropical de terra firme na Floresta Nacional de Caxiuanã (PA). Revista de Ciências Agrárias, 34: 85-106.

Magurran, A.E. 1988. Ecological diversity and its measurement. Princeton University Press, Princeton.

Muniz, F.H.; Cesar, O.; Monteiro, R. 1994. Fitossociologia da vegetação arbórea da Reserva Florestal do Sacavém, São Luís, Maranhão (Brasil). Acta Amazonica, 24(3/4): 219-236.

Noris, K.; McCulloch, N. 2003. Demographic models and the management of endangered species: a case study of the critically endangered Seychelles magpie robin. Journal of applied Ecology, 40: 890-899.

Oliveira, A.N.; Amaral, I.L. 2004. Floristic and phytosociology of a slope forest in Central Amazonia, Amazonas, Brazil. Acta Amazonica, 34(1): 21-34.

Oliver, C.D.; Larson, B.C. 1996. Forest Stand Dynamics. John Wiley \& Sons, New York. 537pp.

Patil, G.P. ; Taillie, C. 1982. Diversity as a concept and its measurement. Journal of the American Statistical Association, 77: 548-567. 
Pereira, N.W.V.; Venturin, N.; Machado, E.L.M.; Scolforo, J.R.S.; Macedo, R.L.G.; D’Oliveira, M.V.N. 2005. Análise das variaçóes temporais na florística e estrutura da comunidade arbórea de uma floresta explorada com plano de manejo. Cerne, 11(3): 263-282.

Phillips, O.L.; Vargas, N.P.; Monteagudo, L.A.; Cruz, P.A.; Zans, C.M.E.; Sánchez, G.W. 2003a. Habitat association among Amazonian tree species: a landscape-scale approach. Journal of Ecology, 91: 757-775.

Phillips, O.L.; Martinez, V.R.; Vargas, N.P.; Monteagudo, L.A.; Zans, C.M.E.; Sánchez, G.W.; Cruz, P.A.; Timana, M.; Yli-Halla, M.; Rose, S. 2003b. Efficient plot-based floristic assessment of tropical forests. Journal of Tropical Ecology, 9(6): 629-645.

Preston, R.J. 1948. North American trees. The Iowa State College Press, Iowa.

Rabinowitz, D. 1981. Seven forms of rarity. In: Synge, H. (ed.) The biological aspects of rare plant conservation. Wiley, New York. p.205-218.

Reynolds, H.T. 1977. The analysis of cross-classifications. Collier Macmillan, New York.

Ricklefs, R.E. 2000. Rarity and diversity in Amazonian forest trees. Trends in Ecology and Evolution, 15(3): 83-84.

Rodriguez, L.C.E. 1998. Monitoramento florestal: iniciativas, definiçōes e recomendações. IPEF, 12(31): 9-21.

Schulman, L. 2003. Nature conservation in Amazonia: the role of biological theory in reserve Delimitation. Lyonia, 5(1): 91100 .
Sist, P.; Saridan, A. 1999. Stand structure and floristic composition of a primary lowland dipterocarp forest in East Kalimantan. Journal of Tropical Forest Science, 11(4): 704-722.

Tilman, D.; Reich, P.B., Knops, J.; Wedin, D.; Moelke, T.; Lehman, C. 2001. Diversity and productivity in a long-term grassland experiment. Science, 294: 843-845.

Tuomisto, H., Ruololainen, K.; Kalliola, R.; Linna, A.; Danjoy, W.; Rodriguez, Z. 1995. Dissecting Amazonian biodiversity. Science, 269: 63-66.

Tuomisto, H., Ruololainen, K.; Yli-Halla, M. 2003. Dispersal, environment, and floristic variation of western Amazonian forests. Science, 299: 241-244.

Uhl, C.; Vieira, I.C.G. 1989. Ecological impacts of selective logging in the Brazilian Amazon: a case study from the Paragominas region in the state of Para. Biotropica, 21: 98-106.

Ward, J.H. 1963. Hierarchical Grouping to Optimize an Objective Function. Journal of the American Statistical Association, 58: 236-244.

Whittaker, R.H. 1972. Evolution and measurement of species diversity: Taxon, 21: 213-251.

Whittaker, R.J.; Willis, K.J.; Field, R. 2001. Scale and species richness: towards a general, hierarchical theory of species diversity. Journal of Biogeography, 28: 453-470.

Recebido em 09/10/2007

Aceito em 17/04/2008 